\title{
Transfer between Pavlovian facilitators and instrumental discriminative stimuli
}

\author{
T. L. DAVIDSON, JUAN APARICIO, and ROBERT A. RESCORLA \\ University of Pennsylvania, Philadelphia, Pennsylvania
}

\begin{abstract}
Two experiments assessed the degree to which Pavlovian facilitators were interchangeable with instrumental discriminative stimuli $\left(S^{d}\right)$ ). In Experiment 1 , rats were trained in a Pavlovian paradigm in which one stimulus (i.e., a facilitator) signaled the reinforcement of another stimulus (i.e., a target). Next, the rats were given instrumental discrimination training in which an $S^{d}$ signaled the reinforcement of barpressing. A transfer test then assessed the capacity of the Pavlovian facilitator to promote barpressing. The results showed that the facilitator promoted significant barpressing, both when it was presented alone and when it was presented in compound with the $S^{d}$. Reliable transfer was not obtained with a "pseudofacilitator" control stimulus that, during training, was uninformative about the reinforcement of its target. Experiment 2 showed that a stimulus trained as an instrumental $S^{d}$ reliably augmented responding to a stimulus previously trained as a target in a Pavlovian facilitation paradigm. A "pseudo-S" that, during training, was uninformative about the reinforcement of barpressing failed to promote such transfer. These results show that Pavlovian facilitators and instrumental $S^{d} \mathbf{s}$ are interchangeable to a significant degree, and suggest that facilitators and $S^{d} s$ may act via similar mechanisms.
\end{abstract}

There has been considerable recent interest in Pavlovian procedures in which one stimulus $(B)$ is informative about the relationship between another stimulus $(A)$ and the unconditioned stimulus (US). For instance, Holland (1983) and Rescorla (1985) have studied paradigms in which $A$ is reinforced when preceded by $B$ but nonreinforced when presented alone. Animals given this $A^{-}$, $B A+$ treatment commonly respond more to $A$ accompanied by $B$ than to $A$ presented alone. A similar outcome is also obtained when the $A-, B A+$ treatment is augmented by separate nonreinforced presentations of $B$ (Davidson \& Rescorla, 1986; Ross \& Holland, 1982). Ross and Holland (1982) have described $B$ as "setting the occasion" for the reinforcement of $A$. Rescorla (1985) has described $B$ as a "facilitator" of responding to $A$.

These procedures are of interest not only for what they might reveal about Pavlovian conditioning, but also for their relationship to certain instrumental learning paradigms. As Holland (1983) and Rescorla (1985) have pointed out, there is a formal similarity between the $B$ stimulus in these Pavlovian paradigms and the discriminative stimulus $\left(\mathbf{S}^{\mathbf{d}}\right)$ in instrumental training paradigms. In the Pavlovian paradigms, only the joint occurrence of $A$ with $B$ is followed by reinforcement; neither $A$ nor $B$ alone is reinforced. Similarly, in operant discriminations, only

This work was supported by Grant BNS-83081 from the National Science Foundation and by Grant T32 MH 17168 from the National Institute of Mental Health. This work was conducted while the first author was a postdoctoral researcher with the Department of Psychology and the Institute of Neurological Sciences at the University of Pennsylvania. The authors thank Marie Snyder-Seaman for typing this manuscript. Requests for reprints should be addressed to Terry Davidson, who is now at the Department of Psychology, Virginia Military Institute, Lexington, VA 24450. the joint occurrence of the $S^{d}$ and the response produces reinforcement. Hence, just as the facilitator of a Pavlovian paradigm signals when a target conditioned stimulus (CS) will be reinforced, the $S^{d}$ of an instrumental paradigm signals when a target response will be reinforced. This parallel is also encouraged by recent discussions (e.g., Colwill \& Rescorla, 1986) that describe simple instrumental learning as involving associations between a response and a reinforcer, and simple Pavlovian conditioning as involving associations between a CS and a reinforcer. This suggests that events associated with reinforcement become targets of both Pavlovian facilitators and instrumental $S^{\mathrm{d}} \mathbf{s}$.

One implication of this analysis is that the mechanism by which facilitators promote responding to Pavlovian CSs may be like that by which $S^{d}$ s promote instrumental responding. If so, facilitators and $S^{d} s$ might be interchangeable in the sense that they might act on each other's targets. Consistent with this possibility, Colwill and Rescorla (1986) reported that, for pigeon subjects, a diffuse noise trained as a Pavlovian facilitator acted in place of an $S^{d}$ to promote a previously trained instrumental treadlepress.

The present experiments provided a more detailed investigation of the interchangeability of facilitators and $S^{d} \mathbf{s}$. In both experiments, rat subjects were trained to perform food-rewarded instrumental barpress responses. Pavlovian conditioning took place in an observational paradigm like that employed by Holland (1977). In Experiment 1, we examined the question of whether a stimulus trained as a facilitator could substitute for an $S^{d}$ to control barpressing. In Experiment 2, we asked whether an $S^{d}$ trained to control barpressing could substitute for a facilitator to promote responding to a Pavlovian CS. 


\section{EXPERIMENT 1}

In this experiment, the degree to which a stimulus trained as a Pavlovian facilitator could control the occurrence of an instrumentally trained response was assessed. The rats were concurrently trained on two discrimination problems. One problem attempted to establish a visual stimulus $(B)$ as a facilitator for a diffuse auditory stimulus $(A)$. The $A$ stimulus was reinforced only when it was preceded by $B$; neither $A$ nor $B$ was reinforced when presented alone. The other problem used a different visual stimulus $\left(B^{\prime}\right)$ in a related pseudofacilitation procedure in which $B^{\prime}$ was treated like $B$ except that $B^{\prime}$ was made uninformative about the reinforcement of another auditory stimulus $\left(A^{\prime}\right)$. This was accomplished by reinforcing $A^{\prime}$ both when it was preceded by $B^{\prime}$ and when it was presented alone. In addition, the $\boldsymbol{B}^{\prime}$ stimulus was presented alone without reinforcement. Davidson and Rescorla (1986) showed that such an $A^{\prime}+, B^{\prime} A^{\prime}+, B^{\prime}$ - discrimination yielded a $B^{\prime}$ that lacked the capacity to facilitate responding to Pavlovian targets. When Pavlovian training was completed, the rats were given discriminative instrumental barpress training with a white noise as the $S^{d}$. We then assessed the capacity of facilitator $B$ and pseudofacilitator $B^{\prime}$ to promote barpressing, both in the presence and in the absence of the $S^{d}$.

\section{Method}

Subjects and Apparatus. The subjects were 16 naive male Sprague-Dawley rats, about 100 days old at the start of the experiment. Food deprivation was used to maintain the rats at $80 \%$ of their ad-lib body weights. They received water ad lib throughout the experiment, except during experimental sessions.

The experimental chambers consisted of four identical $22.9 \times$ $20.3 \times 20.3 \mathrm{~cm}$ boxes. Each chamber had a recessed food magazine in the center of the front wall and a 6-W jeweled lamp above the magazine that provided constant chamber illumination. Additionally, the food magazine itself was dimly illuminated by a neon glow tube. The floor of the chamber was composed of $.48-\mathrm{cm}$ stainless steel rods spaced $1.9 \mathrm{~cm}$ apart. Each chamber was equipped with a retractable response lever. The front and back walls of the chambers were aluminum; the side walls were clear acrylic plastic. Each chamber was enclosed in a light- and sound-resistant shell, equipped with a clear acrylic plastic door to allow for observation of the rats. A 6-W houselight, which served as a flashing $(2-\mathrm{Hz})$ light signal, was mounted on the inside wall of this shell about $10 \mathrm{~cm}$ from the inside wall of the experimental chamber, about $5 \mathrm{~cm}$ above it, and approximately midway between its front and back walls. The houselight was mounted between two 3-in. speakers, one of which served to deliver auditory stimuli. An additional light assembly, which provided a steady light cue, was mounted parallel to and $5 \mathrm{~cm}$ below the outside wall of the experimental chamber. Each assembly consisted of a tubular, 40-W incandescent lamp, $14 \mathrm{~cm}$ in length, contained within a 20 -cm-long prism-shaped housing that was opaque on two sides and translucent on the third side, which faced the chamber. When illuminated, each assembly appeared as an evenly lit $10 \times 20 \mathrm{~cm}$ patch of light. The sound of a ventilating fan attached to the side of the shell served to continuously mask extraneous noise.

The behavior of the rats was monitored on a video recording system. This system consisted of a Panasonic low-light camera (Model WV-250A) mounted $2.4 \mathrm{~m}$ from the experimental chambers so as to include all four chambers in its field of view, an RCA VHS video- cassette recorder, and a 22-in. Panasonic monitor (Model TR-220M).

Procedure. The rats were divided into four squads of 4 animals each. Each rat was assigned to a different experimental chamber and was run in that chamber throughout the experiment. The squads were run in the same order each day.

Each rat was magazine trained with food pellets $(45 \mathrm{mg}, \mathrm{P}$. J. Noyes Co.) automatically delivered on a variable-time (VT) 1 -min schedule. Magazine training terminated following delivery of 10 reinforcements. A reinforcement consisted of two food pellets, delivered one at a time with an interval of $.25 \mathrm{sec}$; the total duration of feeder operation was .5 sec.

Facilitation training began on the day following magazine training. All rats were concurrently trained on two discrimination problems. One problem was of the form LT,$+ \mathrm{L}-, \mathrm{T}-$. On LT+ serial compound trials, a visual facilitator $(L)$ was presented for $15 \mathrm{sec}$. An auditory target stimulus (T) overlapped the final $5 \mathrm{sec}$ of the light; they coterminated in reinforcement $(+)$. On T-trials, a 5-sec target was presented alone and was not reinforced. In addition, 15-sec L - trials were given to ensure low levels of responding to the facilitator alone. The other discrimination problem was of the form $L^{\prime} T^{\prime}+, L^{\prime}-, T^{\prime}+$, where the auditory target stimulus $\mathrm{T}^{\prime}$ was reinforced not only when it was in compound with visual stimulus L', but also when it was presented by itself. $L$ ' was referred to as a "pseudofacilitator" in this problem because it provided no information about the reinforcement of $T$ '. Temporal parameters were the same for both discrimination problems.

The squads were counterbalanced with respect to the identity of the visual stimuli as $L$ and $L^{\prime}$ and with respect to the identity of the auditory stimuli serving as $T$ and $T^{\prime}$. For half of the rats, a flashing houselight served as $\mathrm{L}$ and a steady light served as $\mathrm{L}^{\prime}$. The identities of the $\mathrm{L}$ and $\mathrm{L}^{\prime}$ stimuli were reversed for the remaining half. Similarly, half of the rats had a $1500-\mathrm{Hz}$ tone as $\mathrm{T}$ and a clicker as $T^{\prime}$, with the identities of the $T$ and $T^{\prime}$ stimuli reversed for the remaining half. In addition, half the rats received the tone presented with their $L$ and the clicker presented with their $L^{\prime}$, while the auditory stimuli presented with $\mathrm{L}$ and $\mathrm{L}^{\prime}$ were reversed for the remaining rats.

All rats received three presentations of each of the six trial types during each 48-min session, producing a mean intertrial interval (ITI) of $160 \mathrm{sec}$. Trials were presented in three different quasirandom sequences, with each sequence used once every 3 days. All rats received one training session a day for 26 days.

The behavior of each subject on each trial was videotaped. Learning was assessed via a behavioral observation technique like that described by Davidson and Rescorla (1986). Three observations were made from the videotape for each rat during each 15 -sec trial period. This trial period began with the onset of the facilitator on serial compound trials and on facilitator- and pseudofacilitator-alone trials, and began $10 \mathrm{sec}$ before stimulus onset on target-alone trials. Paced by a $1 / 1.25$-sec auditory signal recorded on the tape, the observer shifted his gaze from chamber to chamber, observing each of the 4 rats once every $5 \mathrm{sec}$ during the trial period. Only one behavior was recorded on each observation. The following six categories of behavior were recorded: (1) Headjerking-short rapid horizontal and/or vertical movements of the head, usually directed toward the food magazine. (2) Rearing-standing on the hindlegs with both forepaws off the grid floor. This category excludes grooming movements performed while the rat was standing on its hindlegs. (3) Magazine-standing motionless in front of the food magazine, with the nose or head close to or in the magazine. (4) Locomotion-movement of all four feet in a forward direction. (5) Magazine-oriented activity-movement of the head and neck oriented toward the food magazine; this movement was slower, and/or traversed a smaller distance, than headjerking. (6) Other--all behaviors other than those described above.

Barpress training began on the day after completion of facilitation training. The response lever was inserted into each operant 
chamber, and each rat was trained to a criterion of 50 responses, with each response being reinforced with one 45-mg Noyes pellet. Discrimination training began the day after all rats had attained this criterion. On the first day of this training, all rats were given 30 presentations of a 15-sec white-noise $S^{d}$, with a mean ITI of $45 \mathrm{sec}$. All barpresses made during the $S^{d}$ on this day were reinforced. Responses made during the ITI were not reinforced. All rats were then given 15 additional days of discrimination training that were the same as that given on the first day except that responses made during the $S^{d}$ were reinforced on a variable-interval 30-sec (VI30) schedule.

All rats were given 6 days of facilitation retraining, beginning the day after the completion of the last barpress discrimination session. Retraining was conducted under the same conditions as original facilitation training, and performance at the end of retraining approximated that observed at the conclusion of original training. On the day following the last session of facilitation retraining, the bar was reinserted into each chamber and all rats were given one session of $S^{d}$ extinction training. This extinction session was given to allow room for augmentation of responding to stimuli presented in compound with the $S^{d}$ during subsequent testing. $S^{d}$ extinction training was conducted in the same manner as original barpress discrimination training, except that all responses were nonreinforced.

Next, we compared the relative capacities of facilitators and pseudofacilitators to promote barpressing. The bar was inserted and all rats were given one test session, which took place 2 days after the session of $S^{d}$ extinction. The test session began with five nonreinforced presentations of the white-noise $S^{d}$, with a mean ITI of $45 \mathrm{sec}$, the same as in discrimination training. This was followed by three blocks of five types of nonreinforced trials: $S^{d}$, facilitator, and pseudofacilitator alone, as well as the $S^{d}$ in compound with the facilitator and pseudofacilitator. The mean ITI was $160 \mathrm{sec}$, as in facilitation training. Stimulus duration on all trials was $15 \mathrm{sec}$.

Beginning the day following this test session, all rats were given an additional four sessions of $S^{d}$ extinction under the conditions described previously. On each of the next 2 days, the ability of the facilitators and pseudofacilitators to promote barpressing was retested. Each session was conducted under the conditions described for the original test

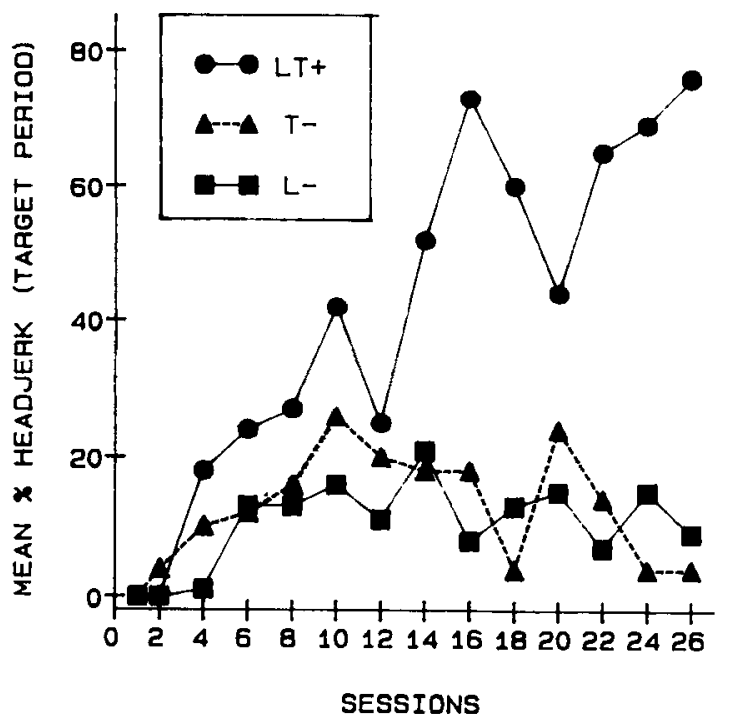

\section{Results and Discussion}

Facilitation training. As reported in other studies that have observed behavior to food-reinforced auditory cues (e.g., Holland, 1977), headjerking emerged as the primary index of conditioning. The left panel of Figure 1 shows the mean percentage of observations scored as headjerking during the last $5 \mathrm{sec}$ for each trial type of the LT+, $\mathrm{L}-, \mathrm{T}-$ discrimination during training. As shown in that figure, the rats came to headjerk more on LT + compound trials than on either $\mathrm{L}-$ or $\mathrm{T}-$ trials. Headjerking was reliably more frequent during $\mathrm{LT}$ than during either $\mathrm{T}$ alone [Wilcoxon $T(14)=0, p<.001]$ or $\mathrm{L}$ alone $[T(14)$ $=.001, p<.01]$ in the final session of training. Differences in headjerking on $\mathrm{L}-$ relative to $\mathrm{T}$ - trials were not reliable

Headjerking over the course of training for the $L^{\prime} T^{\prime}+$, $\mathrm{L}^{\prime}-, \mathrm{T}^{\prime}+$ pseudofacilitation discrimination problem is shown in the right panel of Figure 1. Responding was high during $\mathrm{L}^{\prime} \mathrm{T}^{\prime}+$ compound and during $\mathrm{T}^{\prime}+$ alone trials. Headjerking during the nonreinforced $L^{\prime}-$ trials was at a consistent low level throughout training. Mean percent headjerking was reliably greater on $L^{\prime} T^{\prime}+$ trials than on $\mathrm{L}^{\prime}-$ trials in the last session of training $[T(15)=0$, $p<.001]$. Headjerking on $\mathrm{T}^{\prime}+$ trials was also reliably greater than on $\mathrm{L}^{\prime}-$ trials during the last training session $[T(14)=0, p<.001]$. The difference in headjerking between $L^{\prime} T^{\prime}+$ and $T^{\prime}+$ trials was not significant.

These results show that the rats learned to respond differently to the LT compound than they did to the separately presented $\mathrm{L}$ and $\mathrm{T}$ stimuli in the facilitation problem. Differential responding was not obtained between the $L^{\prime} T^{\prime}$ compound and the separately presented $T^{\prime}$ stimulus in the pseudofacilitation problem. The low amount of headjerking when $\mathrm{L}$ was presented alone indicates that

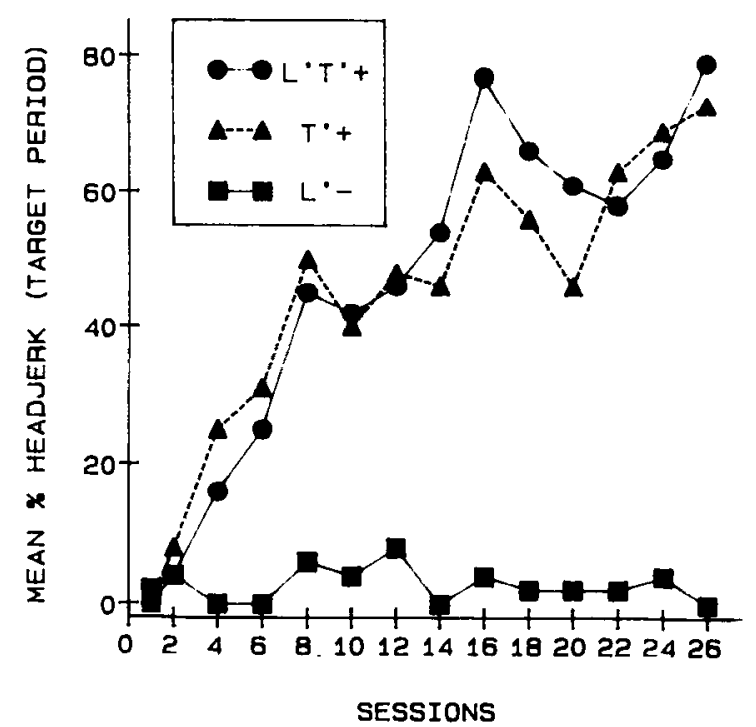

Figure 1. Mean percentage of target-period headjerking during Pavlovian discrimination training in Experiment 1. Responding on the facilitation problem (left panel) is shown to the reinforced serial compound $(\mathrm{LT}+$ ) and to the separate nonreinforced presentations of each its elements ( $T$ - and $L-$ ). Responding on the pseudofacilitation problem (right panel) is shown to the reinforced $L^{\prime} T^{\prime}+$ serial compound and to each of its elements $\left(T^{\prime}+\right.$ and $\left.L^{\prime}-\right)$ when they were presented alone. 
it was not directly associated with reinforcement. It has been suggested (Holland, 1977) that rearing is the predominant form of the conditioned response to foodreinforced visual cues. However, in our laboratory, headjerking, and not rearing, is found to be the conditioned response to visual signals for food (Davidson \& Rescorla, 1986). In the present experiment, mean rearing during target-period observations on $L$ trials was only $4.2 \%$ on the last day of training. Thus, neither headjerking nor rearing provided evidence that $\mathrm{L}$ functioned as a $\mathrm{CS}$.

Instrumental training. Discriminative control of barpressing developed rapidly during the $S^{d}$. On the final day of training, a discrimination ratio of the form $A /(A+B)$, where $A$ is the mean number of responses per minute during the $S^{d}$ and $B$ is the mean number of responses per minute during the ITI, reached a value of .81 .

Transfer testing. Figure 2 shows responding during the facilitator $(\mathrm{L})$ and the pseudofacilitator $\left(\mathrm{L}^{\prime}\right)$ when they were presented alone and in compound with the $S^{d}$. The results are presented in terms of discrimination ratios of the form $A /(A+B)$, as defined above.

The results of the first test session are shown in the left panel. During this test, the discrimination ratio was reliably greater for $\mathrm{L}$ than for $\mathrm{L}^{\prime}$ when the two stimuli were presented alone $[T(13)=10, p<.01]$. A similar pattern emerged, but was not reliable, when $L$ and $L^{\prime}$ were presented in compound with the $S^{d}$. However, combining $L^{\prime}$ with the $S^{d}$ did produce a reliable lower discrimination ratio than did the $S^{\mathrm{d}}$ alone $[T(13)=11, p<.02]$; the ratios for the $S^{d}$ alone and in compound with $L$ were not reliably different. Baseline rates of responding for the $S^{d}, L+S^{d}$, and $L^{\prime}+S^{d}$ were $18.1,17.3$, and 6.75 responses/min, respectively, during the first test session. Consequently, when expressed as discrimination ratios, these results indicate that the pseudofacilitator had a suppressive effect on responding whereas the facilitator did not. However, the absolute levels of barpressing indicate that the facilitator did augment responding. During this test, the mean number of responses per minute during $\mathrm{L}$, $\mathrm{L}^{\prime}$, and the ITI were $2.67,0.83$, and 1.03 , respectively. The rate of responding during $\mathrm{L}$ was reliably greater than that during both the ITI $[T(16)=13, p<.01]$ and the $\mathrm{L}^{\prime}[T(14)=12, p<.01]$. The difference in response rate between $\mathrm{L}^{\prime}$ and the ITI was not reliable.

The right panel of Figure 2 shows the results of the second test, which followed additional extinction to the $S^{d}$ alone. During that test, $L$ and $L^{\prime}$ no longer controlled different discrimination ratios when presented alone. Baseline rates of responding to $L$ and $L^{\prime}$ during the second test session were 1.5 and 0.87 responses $/ \mathrm{min}$, respectively. However, the lowered discrimination ratio to the $S^{d}$ alone permitted the addition of $L$ to show an elevation. The discrimination ratio of the $L+S^{d}$ compound was reliably greater than that to the $L^{\prime}+S^{d}$ compound $[T(11)$ $=10.5, p<.05]$. During this test, the mean number of responses per minute was $7.2,5.3$, and 2.8 to $L+S^{d}, S^{d}$, and $L^{\prime} S^{d}$, respectively. Responding to the $L+S^{d}$ compound was reliably greater than that to the $L^{\prime}+S^{d}$ compound $[T(14)=20, p<.025]$ and that to the $S^{d}$ alone $[T(15)=14, p<.01]$. Responding did not differ when the $S^{d}$ was alone or when it was in compound with the pseudofacilitator.

These results suggest that a stimulus previously trained as a Pavlovian facilitator can augment performance of an

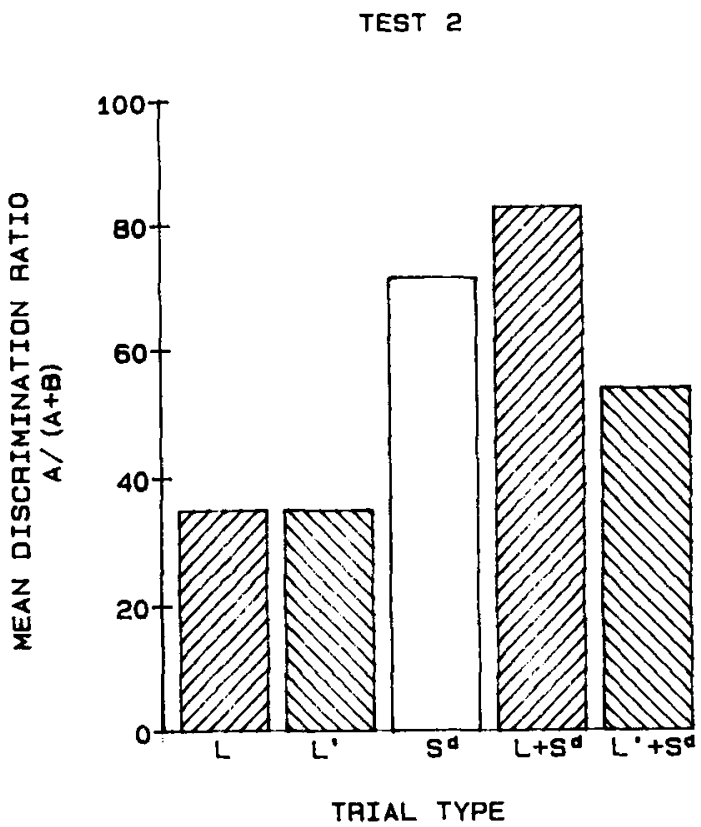

Figure 2. Mean discrimination ratios during the first instrumental barpress test session of Experiment 1 on trials in which the facilitator (L), pseudofacilitator $\left(L^{\prime}\right)$, and $S^{d}$ was each presented alone and on trials in which the $S^{d}$ was presented in compound with the facilitator $\left(L+S^{d}\right)$ and the pseudofacilitator $\left(L^{\prime}+S^{d}\right)$, respectively. 
instrumental response that was previously under the control of an $S^{d}$. The facilitator was found to promote instrumental responding when it was presented alone, and to further elevate responding when combined with the $S^{d}$. Neither of these effects was obtained with the pseudofacilitator control stimulus. Moreover, the suppressive effects of the control stimulus suggest that the facilitator had its effects in the face of substantial stimulus generalization decrement. It is not likely that the response-promoting capacity of the facilitator was dependent on its being directly associated with reinforcement. The facilitator did not appear to function as a CS during original training. Rather, it appeared to modulate the capacity of other stimuli to elicit responding. The results indicate that this modulatory capacity transferred to promote performance of an instrumental response.

\section{EXPERIMENT 2}

The results of Experiment 1 suggest that a stimulus trained as a Pavlovian facilitator can substitute, at least partly, for an $S^{d}$ in promoting the occurrence of an instrumental response. Experiment 2 examined the converse possibility that an $S^{d}$ can transfer its effects to augment performance to a Pavlovian CS.

Rats were trained to make two instrumental responses. One response (RI) was reinforced on a VI schedule only in the presence of a diffuse visual $S^{d}$. The other response (R2) was reinforced in the presence and absence of a different diffuse visual stimulus $\left(\mathbf{S}^{\mathrm{d}^{\prime}}\right)$. The VI schedule in effect for $\mathbf{R} 2$ during this "pseudo- $S$ " " was the same as that in effect for R1 during the $S^{d}$; however, because R2 was reinforced on the VI schedule whether or not the $S^{\mathbf{d}^{\prime}}$ was present, the stimulus was uninformative about the availability of reinforcement. The two visual stimuli served as the instrumental counterparts of the facilitator and pseudofacilitator of Experiment 1. After completion of instrumental training, all rats were trained with a Pavlovian facilitation procedure in which a clicker was followed by food only when it was preceded by a tone facilitator. Finally, we assessed the ability of the $S^{d}$ and $S^{d^{\prime}}$ to augment responding to the clicker.

\section{Method}

Subjects and Apparatus. The subjects were 16 rats of the same description as those in Experiment 1. The apparatus was the same as that used in Experiment 1.

Procedure. The rats were divided into four squads of 4 rats each. Each rat in each squad was assigned to a different experimental chamber and was run in that chamber throughout the experiment. The squads were run in the same order each day.

Each rat was magazine trained as described for Experiment 1. No response manipulanda were present in the chambers during magazine training. On the day following magazine training, chains were inserted into each chamber. Each rat was then trained to a criterion of 50 chainpull responses, with each chainpull being reinforced with one 45-mg Noyes pellet. On the next day, the chains were removed from the operant chambers, the levers were inserted, and each rat was trained to perform the barpress response to the same criterion as that used for chainpulling. Discrimination training began on the day after each rat had attained this criterion.
On half of the sessions, responses in the presence of a 15-sec visual discriminative stimulus $\left(S^{d}\right)$ were reinforced on a VI-30 schedule and responses made in the absence of the $S^{d}$ were nonreinforced. The rats were given $30 S^{d}$ presentations during each 30-min session of $S^{d}$ training, yielding a mean ITI of $45 \mathrm{sec}$. Half the rats were trained with a flashing-light $S^{d}$, and half were trained with a steady-light $S^{\mathbf{d}}$. In addition, for half the rats, the chain was inserted and the lever was retracted during $S^{d}$ training. The lever was inserted and the chain retracted during $S^{d}$ training for the remaining rats. The stimulus (flashing or steady light) that served as the $S^{d}$ was counterbalanced for each response.

All rats were given "pseudo- $S^{\mathbf{d}}$ " training on the remaining half of the sessions. Pseudo- $S^{d}$ training was the same as the $S^{d}$ training described above, except that responses were reinforced on a VI-30 schedule both in the presence and in the absence of the 15-sec visual $S^{d^{\prime}}$. For each rat, the stimulus (flashing or steady light) that was not used as an $S^{d}$ was used as an $S^{d^{d}}$. Similarly, the response (chainpull or barpress) that was not trained with the $S^{d}$ was trained with the $S^{d^{\prime}}$.

Pavlovian facilitation training began after the completion of 22 sessions (11 each with $S^{d}$ and $S^{d^{\prime}}$ ) of instrumental training. All rats were trained on a discrimination problem of the form $\mathrm{TC}+, \mathrm{T}-$, $\mathrm{C}-$. On TC+ serial compound trials, a tone facilitator (T) was presented for $15 \mathrm{sec}$. A clicker target stimulus (C) overlapped the final $5 \mathrm{sec}$ of the tone, and they coterminated in reinforcement (+). On C-trials, a 5-sec target was presented alone and was not reinforced. T - trials were also given to ensure low levels of responding to the 15-sec facilitator when it was presented alone. All rats received six presentations of each of the three trial types during each 48-min session. The mean ITI was $160 \mathrm{sec}$. Trials were presented in three different quasi-random sequences, with each sequence used once every 3 days. All rats received one training session a day for 13 days.

On the day following the last facilitation training session, a transfer test was conducted. This test compared the capacities of $S^{d}$ and $S^{d^{\prime}}$ to promote responding to the clicker. This session began with two presentations of each trial type used in facilitation training (TC+, $\mathrm{T}-, \mathrm{C}-$ ), and concluded with two nonreinforced presentations of each of five types of test trials: $S^{d}$ alone and in compound with $\mathrm{C}, \mathrm{S}^{\mathrm{d}^{\prime}}$ alone and in compound with $\mathrm{C}$, and $\mathrm{C}$ alone. All stimuli retained their original durations (15 sec for the $S^{d}$ and $S^{d^{\prime}}$ and $5 \mathrm{sec}$ for C); on compound trials, $C$ overlapped the final $5 \mathrm{sec}$ of the instrumental stimuli.

Responding to the clicker during acquisition and transfer was assessed using the behavioral observation method described in Experiment 1 . The behavior of each rat was recorded once every $1.25 \mathrm{sec}$ during each 5 -sec target period.

\section{Results and Discussion}

Instrumental training. By the final day of instrumental training, the mean discrimination ratio for the $S^{d}$ was .82. The discrimination ratio for the $S^{d^{\prime}}$ hovered near .5 throughout training. The discrimination ratio for the $\mathbf{S}^{\mathbf{d}^{\prime}}$ was reliably below that of the $S^{d}[T(16)=0, p<.001]$ on the last day of training.

Facilitation training. The mean percent of observations scored as headjerking during the last $5 \mathrm{sec}$ of the last session of facilitation training was 71.9 for TC+ trials, 0.0 for $\mathrm{C}-$ trials, and 22.9 for $\mathrm{T}-$ trials. The difference between $\mathrm{TC}+$ and $\mathrm{C}-$ was statistically reliable $[T(15)=0, p<.001]$, as was the difference between $\mathrm{TC}+$ and $\mathrm{T}-[T(14)=0, p<.001]$. The difference in headjerking between $\mathrm{C}-$ and $\mathrm{T}-$ trials failed to reach significance on the last training session. Thus, the rats showed that they had solved the facilitation problem by 
headjerking more on reinforced facilitator-target compound trials than on nonreinforced presentations of the facilitator or target alone.

Transfer test. The mean percentage of headjerking during the target periods of the transfer test are shown in Figure 3. Those results are shown separately for the $S^{d}$, the $S^{d^{\prime}}$, and the clicker, as well as for the clicker in compound with each of the other stimuli. Responding was close to zero for each of the individual stimuli. In contrast, responding to the clicker was elevated in the presence of the stimuli from the instrumental training procedures. Responding was greater to the compound of the $S^{d}$ and $C$ than to any of the other trial types $[T \mathrm{~s}(12)<9, p s<.02]$. However, the compound $\mathrm{S}^{\mathrm{d}^{\prime}}$ with $\mathrm{C}$ did not produce reliably more responding than did $\mathrm{C}$ alone. These results were validated by a second observer who scored the behavior of each rat once every $5 \mathrm{sec}$ during the target period of each trial of the test phase. This observer also found that headjerking to the $S^{d^{\prime}}$ and $C$ compound was reliably greater than was headjerking to any of the other trial types (all $T s<0$, $p s<.02$ ), and that headjerking to the compound of $S^{d^{\prime}}$ and $\mathrm{C}$ was not reliably different from that observed to $\mathrm{C}$ alone.

These findings suggest that training a stimulus as an instrumental $S^{\mathrm{d}}$ allows it to promote responding to a stimulus trained as a target in a Pavlovian facilitation paradigm. The results of Experiment 2 therefore complement those of Experiment 1 by indicating that a Pavlovian facilitator and an instrumental $S^{d}$ are interchangeable to a significant degree.

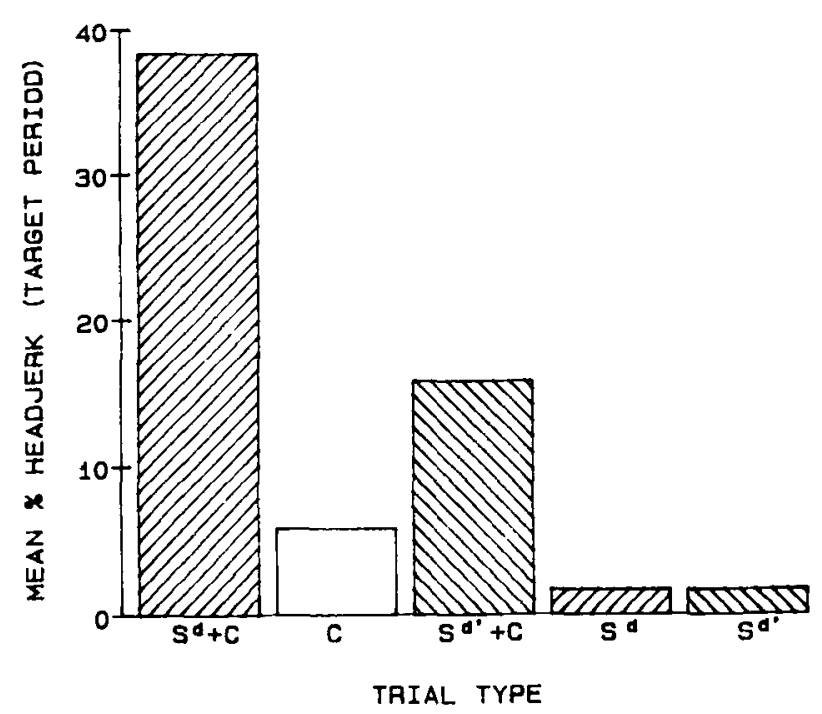

Figure 3. Mean percentage of target-period headjerking during the transfer test in Experiment 2. The figure depicts responding to the Pavlovian target $(C), S^{d}$, and pseudo- $S^{d},\left(S^{d^{\prime}}\right)$ when each was presented alone, and responding to $C$ when it was presented in compound with the $S^{d}\left(S^{d}+C\right)$ and the pseudo- $S^{d}\left(S d^{\prime}+C\right)$, respectively.

\section{GENERAL DISCUSSION}

The results of these experiments demonstrate that Pavlovian facilitators and instrumental $S^{d}$ s share certain functional properties. Experiment 1 found that a facilitator, capable of augmenting performance to a Pavlovian CS, was also capable of augmenting the performance of an instrumental response. Conversely, Experiment 2 found that an instrumental $S^{d}$ trained to increase the performance of an instrumental behavior was also capable of increasing performance to a Pavlovian CS. These results suggest that the logically similar relationships used to establish facilitators and $S^{d}$ s do result in stimuli with similar properties.

This conclusion is supported by several previously reported results. The interaction of Pavlovian facilitators and instrumental $S^{d} \mathbf{S}$ in performance has previously been described with pigeon subjects in a different preparation by Colwill and Rescorla (1986). Additionally, Ross and LoLordo (1986) recently reported that facilitators and $S^{d} s$ interact in the course of learning. They found that a stimulus trained as a Pavlovian facilitator could block the conditioning of another stimulus when the two were jointly used as $S^{d} \mathbf{s}$ in instrumental training. Similarly, a stimulus trained as an $S^{d}$ blocked the conditioning of facilitation to another stimulus in a Pavlovian paradigm. To the degree that blocking of this sort results from shared associative learning, those results imply that facilitators and $\mathbf{S}^{\mathbf{d}} \mathbf{s}$ are closely related in the learning they produce.

Recent theoretical analyses of facilitation have emphasized its difference from Pavlovian excitatory conditioning. Both Holland (1983) and Rescorla (1985) have reported that facilitators trained in an $A-, B A+$ paradigm show little sign of developing substantial excitatory strength. Moreover, such a $B$ stimulus does not change its properties when subjected to standard Pavlovian excitatory and inhibitory training procedures. Furthermore, stimuli deliberately trained as Pavlovian excitors will not act as substitutes for facilitators when tested for their ability to augment responding to target CSs. The results of the present experiments are in line with those observations. In neither experiment did the facilitators show evidence of controlling substantial Pavlovian excitation. Moreover, the $S^{d}$ of Experiment 2 gave little indication of controlling headjerking on its own. Instead, each of these stimuli seemed to require the presence of a target event to exhibit its power. Consequently, although it has been previously reported that simple Pavlovian excitors can sometimes augment instrumental performance (e.g., Bolles \& Grossen, 1970; Estes, 1943; but see Azrin \& Hake, 1969; Mackintosh, 1983, pp. 104-106), it seems unlikely that the facilitators and $S^{d} S$ of the present experiments control instrumental responding by virtue of having excitatory strength.

The present results may also provide some information about the manner in which facilitators act. Both Holland 
(1983) and Rescorla (1985) have argued that facilitators act by modulating the associative effectiveness of target stimuli. However, they have suggested somewhat different points at which this modulation takes place. Holland (1983) has suggested that the facilitator acts on the association between a CS and US, whereas Rescorla (1985) has argued that it acts on the US representation itself. One way in which these accounts differ is in their expectations about the ability of facilitators to transfer to other targets. If the facilitator acts on a particular CS-US association, it might show only limited transfer to other stimuli and responses associated with that same US. On the other hand, if the action of a facilitator is on the US itself, such transfer might be highly successful. Although Holland (1986) has reported failure of such transfer under some circumstances, Davidson and Rescorla (1986) have recently reported highly successful transfer of a facilitator to another Pavlovian target. The results of the present experiments extend that transfer to quite a different kind of target-an instrumental response associated with the same US. That extension is of special importance, because it is less susceptible than some previous instances of transfer to an interpretation in terms of generalization among the training and testing target events. Thus, the present results both extend the action of facilitators to instrumental responding and provide some information about the mechanism by which facilitation acts.

\section{REFERENCES}

AzrIn, N. H., \& HAKE, D. (1969). Positive conditioned suppression: Conditioned suppression using positive reinforcers as the unconditioned stimuli. Journal of the Experimental Analysis of Behavior, 12 , 167-173.
BoLles, R. C., Grossen, N. E. (1970). The noncontingent manipulation of incentive motivation. In J. H. Reynierse (Ed.), Current isswes in animal Learming (pp. 143-174). Lincoln: University of Nebraska Press.

Colwill, R. M., Rescorla, R. A. (1986). Associative structures in instrumental learning. In G. H. Bower (Ed.), The psychology of learning and motivation (Vol. 20, 55-104). New York: Academic Press.

DAvidson, T. L., \&escorla, R. A. (1986). Transfer of facilitation in the rat. Animal Learning \& Behavior, 14, 380-386.

Estes, W. K. (1943). Discriminative conditioning: I. A. discriminative property of conditioned anticipation. Joumal of Experimental Psychology, 32, 150-155.

Holland, P. C. (1977). Conditioned stimulus as a determinant of the form of the Pavlovian conditioned response. Journal of Experimental Psychology: Animal Behavior Processes, 3, 77-104.

Holland, P. C. (1983). Occasion-setting in Pavlovian feature positive discriminations. In M. L. Commons, R. J. Herrnstein, \& A. R. Wagner (Eds.), Quantitative analyses of behavior: Discrimination processes (Vol. 4, pp. 183-206). New York: Ballinger.

Holland, P. C. (1986). Transfer after serial feature positive discrimination training. Leaming \& Motivation, 17, 243-268.

Mackintosh, N. J. (1983). Conditioning and associative learning. New York: Oxford University Press.

Rescorl.A, R. A. (1985). Facilitation and inhibition. In R. R. Miller \& N. E. Spear (Eds.), Information processing in animals: Conditioned inhibition (pp. 299-326). Hillsdale, NJ: Erlbaum.

Ross, R. T., Holland, P. C. (1982). Serial positje patterning: Implications for "occasion setting." Bulletin of the Psychonomic Society, 19, 159-162.

Ross, R. T., LoLordo, V. M. (1986). Evaluation of the relation between Pavlovian occasion-setting and instrumental discriminative stimuli: A blocking analysis. Journal of Experimental Psychology: Animal Behavior Processes, 13, 3-16.

(Manuscript received June 26, 1987; revision accepted for publication December $21,1987$. ) 